\title{
MENSURAÇÃO DA ATENÇÃO DIRIGIDA DO ENFERMEIRO: COMPARAÇÃO ENTRE TRÊS MÉTODOS PSICOFÍSICOS
}

\author{
Edinêis de Brito Guirardello* \\ Fátima Aparecida Emm Faleiros Sousa**
}

GUIRARDELLO, E.de B.; SOUSA, F.A.E.F. Mensuração da atenção dirigida do enfermeiro: comparação entre três métodos psicofísicos. Rev.latino-am.enfermagem, Ribeirão Preto, v. 8, n. 3, p. 108-114, julho 2000.

O grau de atenção dirigida requerido pelo enfermeiro que atua em unidades de cuidados críticos foi avaliado e comparado com o uso de três métodos psicofisicos diferentes: estimativa de magnitudes, estimativa de categorias e estimativa de postos. A amostra constituiu-se de 29 participantes com idades entre 25 a 44 anos. Os dados permitiram concluir que: a) houve concordância significativa entre os participantes para as diferentes situações, com coeficiente de Kendall (W) igual a 0,83 ( $p<0,0016)$; b) os resultados obtidos com o escalonamento de estimativa de magnitudes confirmam a superioridade desse tipo de escalonamento quando comparado aos demais; c) o contínuo de atenção dirigida (não métrico) possui características de contínuo protético.

UNITERMOS: métodos, psicofisica, atenção, enfermagem

\section{INTRODUÇÃO}

A pesquisa em enfermagem está ocupando uma dimensão cada vez maior, na medida em que se busca compreender os fenômenos relacionados à profissão. Um dos maiores obstáculos em conduzir estudos nesta área, usando modelos científicos, é a complexidade do principal elemento de investigação - o ser humano. Esse problema torna-se mais complexo quando as pesquisas estão relacionadas a atitudes ou a comportamento humano, pois cada ser humano é singular quanto à personalidade, à ética, ao ambiente social, aos valores e ao estilo de vida.

Os pesquisadores em saúde e, especificamente em enfermagem, lidam com muitos fenômenos, incluíndo estímulos e sensações. De acordo com MEEK et al. (1992), os julgamentos e interpretações de um dado estímulo e a sensação associada com este variam enormemente de pessoa a pessoa, de tempo a tempo e de instrumento a instrumento.

A estimação de magnitude, é um dos métodos psicofísicos que vem sendo utilizado para mensurar estímulos dessa natureza, ou seja, atributos sociais e clínicos e mostra-se promissora em fornecer para a enfermagem um método de escalonamento de medidas precisas para fenômenos subjetivos. Alguns pesquisadores (STEVENS, 1957, 1975; LODGE, 1981; MEEK et al., 1992; FALEIROS SOUSA \& Da SILVA, 1996a, 1996b; FALEIROS SOUSA, 1999) sugerem que o escalonamento de estimação de magnitude é superior ao escalonamento de categorias que envolve julgamentos da intensidade ou força de um estímulo ou sensação.

A estimação de magnitude é originada de estudos teórico e empírico na psicofísica, envolvendo a relação entre estímulo e resposta (STEVENS, 1957, 1966, 1975). Esta metodologia foi adaptada para os estudos de atitudes por cientistas comportamentais, principalmente por HAMBLIN (1974) e LODGE (1981). A característica desse método que o torna superior a muitos outros métodos de escalonamento para investigação de fenômenos subjetivos inclui a capacidade de eliminar erros aleatórios de medidas e o uso de julgamentos proporcionais em vez de ordenações de posições, de tal forma que relações não-lineares entre variáveis podem ser testadas. LODGE (1981) acrescenta que para garantir que os julgamentos proporcionais ocorram, é necessário que o participante receba treinamento de como fazer julgamento proporcional e se esses procedimentos forem seguidos, não existe razão pela qual as escalas de estímulos sociais não se comportariam como uma lei, como faz com as escalas para estímulos físicos.

Os primeiros estudos em enfermagem que se

\footnotetext{
* Enfermeira. Doutor em Enfermagem. Professor do Departamento de Enfermagem da Faculdade de Ciências Médicas da Universidade Estadual de Campinas - UNICAMP

** Professor Associado junto ao Departamento de Enfermagem Geral e Especializada da Escola de Enfermagem de Ribeirão Preto da Universidade de São Paulo
} 
preocuparam em mensurar fenômenos subjetivos, utilizando o método de estimação de magnitude, foram realizados por HINSHAW \& FIELD (1974) cujo objetivo foi investigar o processo de avaliação dentro de um grupo de enfermeiros e por HINSHAW \& OAKES (1977) que teve como objetivo mensurar atitudes dos pacientes, enfermeiros e médicos sobre suas expectativas em relação à qualidade dos cuidados de enfermagem.

Outros estudos mais recentes realizados por FALEIROS SOUSA (1993); FALEIROS SOUSA \& Da SILVA (1996a, 1999a, 1999b) tiveram como objetivo escalonar o prestígio social do enfermeiro. FALEIROS SOUSA (1997), em sua tese de livre docência, apresentou estudos envolvendo mensurações de atributos sociais e clínicos, quais sejam: mensurações da percepção social do enfermeiro, do reajustamento social, da gravidade dos quadros clínicos e quadro clínico-cirúrgicos, escalonados pelo método de estimação de magnitude e demonstrou que os atributos acima mensurados fornecem escalas em nível de razão confiáveis, válidas e consistentes. (Para outros detalhes ver FALEIROS SOUSA et al.,1998; KAMIZAKI et al., 1998).

De maneira geral, os instrumentos de mensuração utilizados em enfermagem têm gerado apenas dados descritivos ou em nível de mensuração ordinal, nos quais apenas a ordenação entre os valores escalares é admissível. Sendo assim, este estudo propõe-se escalonar, em nível de razão, as situações que requerem atenção dirigida do enfermeiro que trabalham em unidades de cuidados críticos. Essas situações são escalonadas avaliando-se o grau de atenção dirigida do enfermeiro para cada situação através de diferentes métodos psicofísicos.

A atenção dirigida é definida como sendo aquela em que o indivíduo necessita exercer um esforço mental para manter-se diante de um estímulo ou situação. Para JAMES (1950) a atenção dirigida requer que o indivíduo desconsidere alguns objetos ou pensamentos decorrentes tanto do ambiente interno como do ambiente externo para se fixar em uma informação necessária. Segundo CIMPRICH (1992, 1993), a atenção dirigida é crucial na vida do dia a dia, uma vez que permite atividades de planejamento e de resolução de problemas visando um objetivo. Entretanto, os mecanismos inibitórios de atenção dirigida algumas vezes tornam-se sobrecarregados e assim a fadiga de atenção ocorre, levando o indivíduo à distração e à incapacidade de dirigir atenção para outras atividades do dia a dia. Segundo KAPLAN \& KAPLAN (1982) e KAPLAN (1983) a incapacidade para dirigir atenção para outras atividades pode resultar em impaciência, irritabilidade e impulsividade.

$\mathrm{Na}$ sua prática diária, o enfermeiro de unidades de cuidados críticos depara-se com uma grande variedade de estímulos enquanto presta assistência aos pacientes e seus familiares. Esses estímulos podem requerer do enfermeiro um alto grau de atenção dirigida que podem ser identificados como sobrecarga de trabalho, dificuldades relacionadas com a assistência ao paciente criticamente doente, conflito com outros colegas e barulho excessivo (GUIRARDELLO, 1993). Através do escalonamento em nível de razão, é possível identificar e quantificar quais situações requerem mais atenção dirigida.

É possível também verificar se o contínuo de atenção dirigida tem características protéticas ou metatéticas. Segundo STEVENS (1957); STEVENS \& GALANTER (1957) o contínuo de percepções divide-se em duas classes gerais e a natureza dessa divisão é baseada na forma tradicional de distingüir qualidade de quantidade. O contínuo que tem a ver com quantidade é denominado de um contínuo protético e o contínuo que tem a ver com qualidade é denominado de um contínuo metatético. A distinção entre esses dois tipos de contínuos é fundamental, pois eles parecem seguir leis gerais diferentes. Quando as magnitudes subjetivas de um contínuo metatético são colocadas em coordenadas logarítmicas, em função de suas intensidades físicas, observa-se que a relação obedece à lei logarítmica (STEVENS, 1975). Quando as magnitudes de um contínuo protético são projetadas em coordenadas logarítmicas, em função de suas intensidades físicas, observa-se que a relação segue a lei de Stevens ou lei de potência (ENGELMANN, 1966).

\section{OBJETIVO}

Neste estudo, o grau de atenção dirigida do enfermeiro foi avaliado com o uso de três métodos psicofísicos diferentes: estimativa de magnitudes (EM), estimativa de categorias (EC) e estimativas de postos (EP). Os objetivos foram (1) verificar se as ordenações das situações de atenção dirigida derivadas de ambos os métodos psicofísicos são similares entre si; (2) verificar se a variabilidade das estimativas de magnitudes e de categorias é uma função linear das médias geométricas dessas estimativas e (3) verificar mediante comparação entre as estimativas de magnitudes e estimativas de categorias, se o contínuo não métrico de atenção dirigida possui características protéticas ou metatéticas. Em todos os casos, quando se fala em atenção dirigida, refere-se à atenção vivenciada ou percebida pelo enfermeiro, ou seja, a percepção que o enfermeiro tem da atenção requerida.

\section{METODOLOGIA}

O instrumento consiste em 15 situações 
habitualmente vivenciadas pelos enfermeiros atuantes em unidades de cuidados críticos (Quadro 1), resultantes de estudo realizado por GUIRARDELLO (1993). Foram selecionadas as 15 situações com maiores médias aritméticas, significando as situações consideradas no estudo citado como os que requerem maior grau de atenção dirigida, julgadas pelo método de estimação de categorias. Após definição operacional foram posteriormente submetidas a procedimentos de validação aparente e de conteúdo (participaram seis juizes) e teste piloto (participaram oito enfermeiros, os quais não foram excluídos da amostra, uma vez que não houve modificações no instrumento de coleta de dados).

\section{Quadro 1 - Situações com maiores médias aritméticas}

01. Diversas tarefas de enfermagem a serem completadas 02. Aumento do fluxo de pessoas na sua área de trabalho 03. Suporte a familiares com necessidade de atenção do ponto vista emocional

04. Observação do sofrimento de um paciente

05. Muitos equipamentos na área de trabalho

06. Desenvolvimento de procedimentos que são dolorosos para o paciente

07. Alto nivel de barulho na unidade

08. Muitas decisões rápidas a serem tomadas

09. O médico responsável pelo paciente deixa de transmitir informação a respeito da condição de saúde do paciente

10. Tempo insuficiente para dar suporte emocional para o paciente

11. Area de trabalho muito confusa

12. Tempo insuficiente para completar todas as suas tarefas

13. Cuidar de paciente que necessita de suporte emocional

14. Número insuficiente de pessoas de enfermagem para prestar assistência a pacientes de alta complexidade

15. Imprevistos com o pessoal de enfermagem e escala de plantão

\subsection{Participantes}

A amostra constituiu-se de 29 participantes, sendo a maioria do sexo feminino (26 mulheres e 2 homens), atuantes em unidades de cuidados críticos de um hospital universitário, com idades entre 25 a 44 anos e tempo médio de experiência na área de seis anos. Todos os participantes estavam tendo contato pela primeira vez com este tipo de experimento.

\subsection{Material}

Foram elaborados três blocos de papel, contendo na primeira página instruções específicas para cada tipo de método pscofísico. Para os métodos de estimação de magnitudes e estimação de categorias, as 15 situações foram apresentadas uma a uma, em cartões. No método de estimação de postos, uma lista das 15 situações foi anexada às instruções específicas para este método.

\subsection{Procedimento}

O grau de atenção dirigida requerido pelo profissional enfermeiro foi avaliado com o uso de três métodos psicofísicos diferentes: estimativa de magnitudes (escala de razão), estimativa de categorias (escala intervalar) e estimativa de postos (escala ordinal). No método de estimação de magnitudes, seguindo o protocolo descrito por HINSHAW (1978) e LODGE (1981), a tarefa dos participantes foi assinalar um número a cada situação que fosse proporcional à quantidade de atenção dirigida requerida às mesmas em relação ao módulo padrão previamente determinado pelo pesquisador. Dessa forma, se o participante julgasse que uma dada situação requeresse duas vezes mais atenção dirigida do que o módulo padrão, deveria assinalar a ela um número duas vezes maior e assim por diante. As diferentes situações foram apresentados em duas séries de quinze, as quais foram dispostas uma a uma em cartões, numa ordem totalmente aleatória para cada participante. Cada participante então, recebeu instruções escritas da definição operacional para cada um dos conceitos e uma lista de situações. Também receberam instruções de como fazer julgamento proporcional antes de responder o instrumento, o qual foi administrado pelo pesquisador.

No método de estimação de categorias, a tarefa dos participantes foi assinalar uma pontuação de 1 a 7 a cada situação apresentada, em função da atenção dirigida requerida. Os participantes foram orientados a designar o valor máximo de 7 à situação de maior atenção dirigida, e o valor mínimo de 1 à situação de menor atenção dirigida.

No método de estimação de postos, a tarefa dos participantes foi assinalar a cada situação vivenciada um posto em função da quantidade de atenção dirigida requerida. Os participantes foram instruídos a colocar em primeiro lugar (posto 1) a situação de maior atenção dirigida, e em décimo quinto (posto 15) a situação de menor atenção dirigida. Todos os 29 enfermeiros participaram dos três métodos psicofísicos, sendo estes apresentados a cada um na seguinte ordem: i) estimação de magnitudes, ii) estimação de categorias e iii) estimação de postos. Os experimentos foram realizados no próprio local de trabalho de cada participante e os encontros aconteceram em horários que fossem conveniente para $\mathrm{o}$ mesmo, sendo respeitadas as peculiaridades de cada unidade de trabalho.

Todos os participantes receberam instruções escritas sobre o estudo, tendo sido sua participação de forma voluntária e foram orientados que, mesmo após concordar em participar do estudo, poderiam interromper o preenchimento do instrumento a qualquer momento. 


\section{RESULTADOS E DISCUSSÃO}

Os resultados deste Experimento estão apresentados na Tabela 1, na qual estão demonstradas as médias geométricas das estimativas de magnitudes e médias aritméticas das estimativas de categorias e estimativas de postos de cada situação julgada pelos 29 participantes. Também estão apresentadas as ordenações das posições dos julgamentos das situações derivadas de cada método psicofísico.

Pode-se observar que, independente do método psicofísico utilizado, as situações vivenciadas pelos enfermeiros como: 'muitas decisões rápidas a serem tomadas', 'diversas tarefas de enfermagem a serem completadas', 'número insuficiente de pessoas de enfermagem para prestar assistência a pacientes de alta complexidade' e 'observação do sofrimento de um paciente', foram julgadas por requererem maior quantidade de atenção dirigida, enquanto as situações 'muitos equipamentos na área de trabalho', 'aumento do fluxo de pessoas na sua área de trabalho' e 'alto nível de barulho na unidade' foram julgadas por requererem menor quantidade de atenção dirigida.

O coeficiente de concordância de Kendall (W), corrigido para empates, obtido a partir das ordenações das estimativas de magnitudes, das estimativas de categorias e das estimativas de postos, foi igual a 0,83 $(p<0,0016)$, ou seja, resultou em uma concordância significativa entre as ordenações para os diferentes julgamentos dos participantes. Por exemplo, a situação 'tempo insuficiente para dar suporte emocional ao paciente' ocupa a sétima posição e a situação 'muitos equipamentos na área de trabalho' ocupa a décima quinta posição nos três tipos de contínuos de respostas. A análise que se segue foi feita considerando as estimativas médias apresentadas na Tabela 1.

Tabela 1 - Média geométrica das estimativas de magnitudes (EM), média aritmética das estimativas de categorias (EC), média aritmética das estimativas de postos (EP) e ordenação das posições (OP) das situações de atenção dirigida para cada método

\begin{tabular}{|c|c|c|c|c|c|c|}
\hline SITUAÇÕES & $\mathbf{E} \mathbf{M}$ & OP & $\mathbf{E C}$ & OP & $\mathbf{P}$ & oP \\
\hline 01. Diversas tarefas de enfermagem a serem completadas & 234,36 & 3 & 5,86 & 3 & 6,31 & 4 \\
\hline 02. Aumento do fluxo de pessoas na sua área de trabalho & 113,23 & 13 & 4,21 & 14 & 11,24 & 14 \\
\hline $\begin{array}{l}\text { 03. Suporte a familiares com necessidades de atenção do ponto } \\
\text { de vista emocional }\end{array}$ & 142,35 & 11 & 4,93 & 11 & 8,52 & 11 \\
\hline 04. Observação do sofrimento de um paciente & 175,23 & 5 & 5,62 & 5 & 6,10 & 3 \\
\hline 05. Muitos equipamentos na área de trabalho & 82,51 & 15 & 3,93 & 15 & 11,79 & 15 \\
\hline $\begin{array}{l}\text { 06. Desenvolvimento de procedimentos que são dolorosospara } \\
\text { opaciente }\end{array}$ & 166,10 & 6 & 5,13 & 9 & 7,17 & 6 \\
\hline 07. Alto nivel de barulho na unidade & 134,97 & 12 & 4,48 & 13 & 9,21 & 12 \\
\hline 08. Muitas decisões rápidas a serem tomadas & 202,93 & 4 & 6,41 & 1 & 4,72 & 1 \\
\hline $\begin{array}{l}09.0 \text { médico re sponsável pelo paciente deixa de transmitir } \\
\text { informação a respeito da condição de saúde do paciente }\end{array}$ & 100,00 & 14 & 5,52 & 6 & 5,90 & 2 \\
\hline $\begin{array}{l}\text { 10. Tempo insuficiente para dar suporte emocional para o } \\
\text { paciente }\end{array}$ & 161,50 & 7 & 5,24 & 7 & 7,79 & 7 \\
\hline 11. Area de trabalho muito confusa & 265,64 & 1 & 5,72 & 4 & 8,17 & 9 \\
\hline 12. Tempo insuficiente para completar todas as suas tarefas & 148,11 & 9 & 4,90 & 12 & 9,52 & 13 \\
\hline 13. Cuidar do paciente que necessita de suporte emocional & 149,05 & 8 & 4,96 & 10 & 8,41 & 10 \\
\hline $\begin{array}{l}\text { 14. Número insuficiente de pessoas de enfermagem paraprestar } \\
\text { assistência a pacientes de alta complexidade }\end{array}$ & 234,62 & 2 & 6,38 & 2 & 7,10 & 5 \\
\hline $\begin{array}{l}\text { 15. Imprevistos com o pessoal de enfermagem e escala de } \\
\text { plantão }\end{array}$ & 144,76 & 10 & 5,21 & 8 & 8,03 & 8 \\
\hline
\end{tabular}


Diante do exposto, verifica-se que as ordenações obtidas das estimativas resultantes dos três métodos são concordantes para as diferentes situações vivenciadas pelos participantes. Entretanto, existem algumas diferenças fundamentais nos escalonamentos obtidos. No método de estimação de magnitudes, por exemplo, é possível estabelecer as ordenações, as diferenças e principalmente as razões entre os graus de atenção dirigida das diferentes situações. No método de estimação de categorias, é possível estabelecer apenas as ordenações e as diferenças entre os graus de atenção dirigida. Já no método de estimação de postos, pode-se obter apenas uma ordenação das situações em termos de atenção dirigida requerida.

Nos métodos de estimação de categorias e estimação de postos, não é possível conhecer as razões entre os graus de atenção dirigida, ou seja, não é possível saber o quanto o grau de atenção dirigida requerida para uma determinada situação é maior ou menor em comparação ao grau de atenção dirigida requerida de outra situação. Por exemplo, com os dados apresentados na Tabela 1, referentes às estimativas de magnitudes, podemos afirmar que a atenção dirigida requerida para 'diversas tarefas de enfermagem a serem completadas' $(\mathrm{EM}=243,36)$ é quase duas vezes maior que o grau de atenção dirigida requerida para 'tempo insuficiente para completar todas as suas tarefas' $(\mathrm{EM}=148,11)$, ou aproximadamente duas vezes maior que o grau de atenção dirigida requerida para 'cuidar do paciente que necessita de suporte emocional' $(E M=149,05)$, como também afirmar que é aproximadamente três vezes maior que o grau de atenção dirigida requerida para 'muitos equipamentos na área de trabalho' $(\mathrm{EM}=82,51)$.

Para verificar se o contínuo de atenção dirigida possui características protéticas ou metatéticas, como ocorre com os estímulos sensoriais aditivos, as médias aritméticas das estimativas de categorias foram projetadas em função das médias geométricas das estimativas de magnitudes. Em coordenadas lineares, a relação entre essas duas estimativas mostrou uma leve concavidade descendente (Figura 1). Quando as médias aritméticas das estimativas de categorias foram projetadas em função dos logarítmos das médias geométricas das estimativas de magnitudes, a relação mostrou uma concavidade ascendente (Figura 2). De acordo com as Figuras 1 e 2, podemos verificar que o contínuo do grau de atenção dirigida para as diferentes situações vivenciadas pelo profissional enfermeiro é um contínuo que possui características protéticas.

Outro dado interessante é a relação entre os desvios-padrão da média geométrica e as médias geométricas das estimativas de magnitudes (Figura 3). Observa-se que a relação é linear, de maneira que quanto maior a estimativa de magnitude, tanto maior é o desvio padrão da média. A representação desses dados apresentada na Figura 3, confirma o princípio geral conhecido como Lei de Ekman, em que a variabilidade das estimativas é uma função linear da magnitude das estimativas (STEVENS, 1966, 1975). Já a relação entre os desvios-padrão das médias aritméticas das estimativas de categorias e as médias aritméticas das estimativas de categorias podem ser ajustadas por uma reta com inclinação negativa, porém a dispersão dos pontos é muito grande (Figura 4). Entretanto, o usual é uma curva em forma de ' $U$ ' invertida, sendo que as categorias centrais têm maiores desvios e as extremas menores desvios. As pessoas não tem dúvidas nas categorias extremas, mas sim nas intermediárias. De qualquer forma esta relação não segue a lei de Ekman.

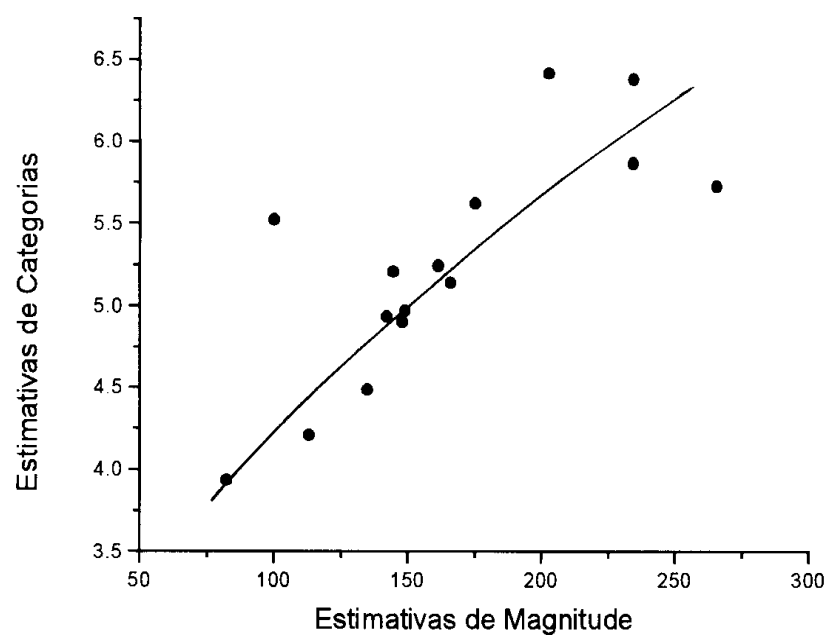

Figura 1 - Relação entre as médias aritméticas das estimativas de categorias e as médias geométricas das estimativas de magnitudes

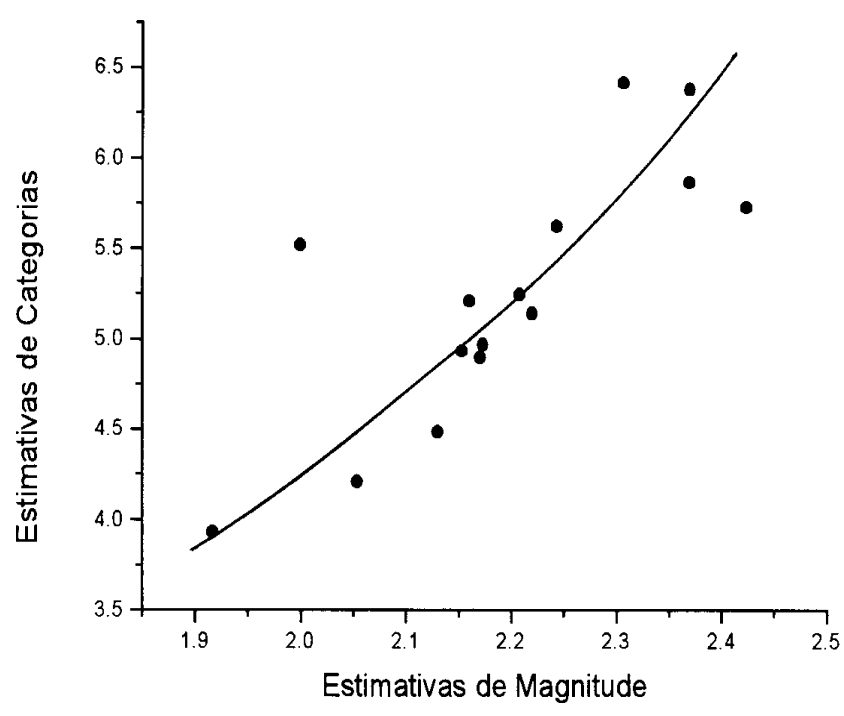

Figura 2 - Relação entre as médias aritméticas das estimativas de categorias e os logaritmos das médias geométricas das estimativas de magnitudes 


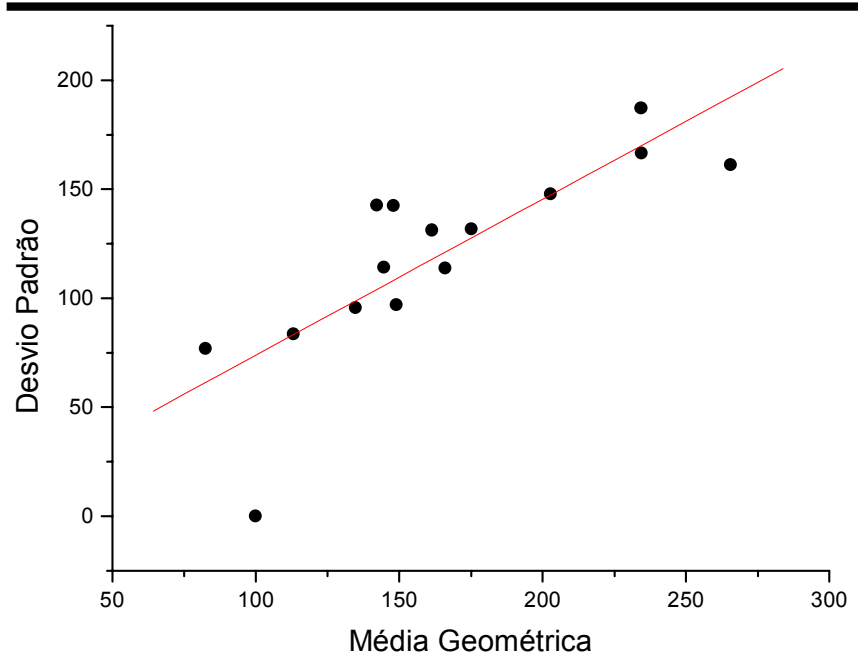

Figura 3 - Relação entre os desvios padrões e as médias geométricas das estimativas de magnitudes

\section{CONSIDERAÇÕES FINAIS}

De acordo com os resultados obtidos nesse Experimento, podemos concluir que o enfermeiro realizou julgamento proporcional e que a escala de razão do grau de atenção dirigida é confiável, válida e estável. Foi significante a concordância entre os três métodos psicofísicos e, adicionalmente, os dados mostraram a

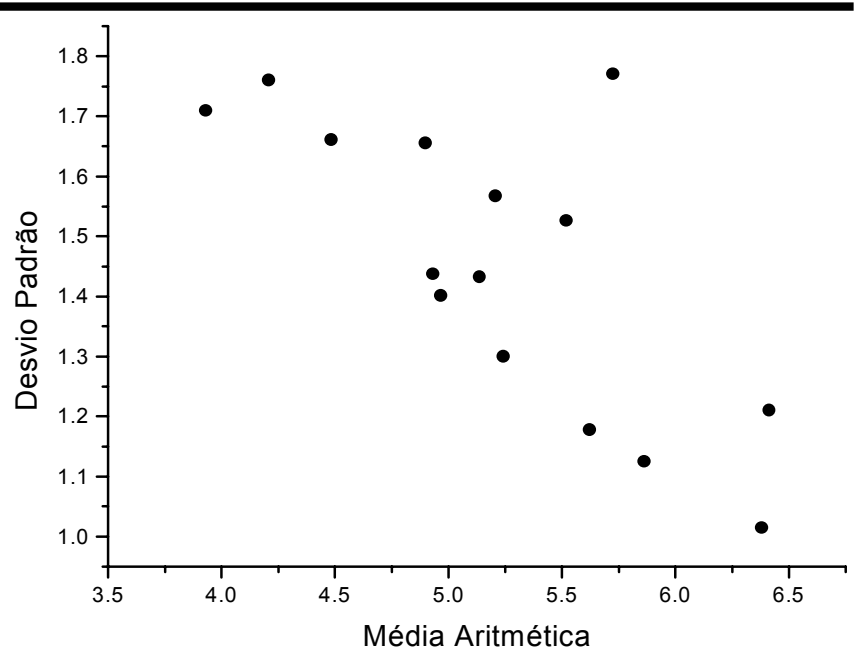

Figura 4 - Relação entre os desvios padrão e as médias aritméticas das estimativas de categorias

vantagem de se utilizar a escala de razão para o julgamento dessas situações, pois no escalonamento de magnitude é possível estabelecer as ordenações, as diferenças e as razões entre os graus de atenção dirigida das diferentes situações, enquanto que no escalonamento de categoria é possível estabelecer apenas as ordenações e as diferenças entre os graus de atenção dirigida.

\section{MEASUREMENT OF NURSES DIRECTED CARE: COMPARISON AMONG THREE PSYCHOPHYSICS METHODS}

The degree of directed care required from nurses who work at the critical care unit environment was assessed and compared through three different psychophysical methods: magnitude estimation, category rating and ordinal scales. The sample was formed by 29 participants whose ages varied from 25 to 44 years. Based on the results, authors concluded that: a) data indicated a high concordance among participants regarding different situations with a Kendall's coefficient of $0.83(p<0.0016)$; b) the results obtained with magnitude estimation scaling proved superiority of this method when compared to others. c) the continuum of directed attention (non metric) has characteristics of a protetic continuum.

KEY WORDS: measurement, psychophysics, nurses directed attention

\section{MEDICIÓN DE LA ATENCIÓN DIRECTA DEL ENFERMERO: COMPARACIÓN ENTRE TRES MÉTODOS PSICOFÍSICOS}

El grado de atención directa requerido por el enfermero que actúa en unidades de cuidados críticos fue evaluado y comparado con el uso de tres métodos psicofisicos diferentes: estimativa de magnitudes, estimativa de categorías y estimativa de puestos. La muestra se constituyo con 29 participantes de edades entre 25 a 44 años. Los datos permitieron concluir que: a) hubo concordancia significativa entre los participantes para las diferentes situaciones, cuyo coeficiente de kendall (W) igual a 0,83 $(p<0,0016)$; b) los resultados obtenidos con el escalonamiento de estimativa de magnitudes confirman la superioridad de ese tipo de escalonamiento cuando se compara con los demás; c) el continuo de atención directa (no métrico) posee características de protético continuo. 


\section{REFERÊNCIAS BIBLIOGRÁFICAS}

01. CIMPRICH, B. Attentional fatigue following breast cancer surgery. Res. Nurs. \& Health, v. 15, p. 199-207, 1992.

02. CIMPRICH, B. Development of an intervention to restore attention in cancer patients. Cancer Nurs., v. 16, n. 2, p. 83-92, 1993.

03. ENGELMANN, A. A lei de potência de Stevens: um caso de constância perceptiva? J. Bras. Psicol., v. 3, p. 19-48, 1966.

04. FALEIROS SOUSA, F.A.E. Prestígio profissional do enfermeiro: um enfoque da psicofísica social. Ribeirão Preto, 1993. 197p. Tese ( Doutorado) Escola de Enfermagem de Ribeirão Preto, Universidade de São Paulo.

05. FALEIROS SOUSA, F.A.E. Métrica do consenso social e clínico: um enfoque experimental. Ribeirão Preto, 1997. 263p. Tese (LivreDocência) - Escola de Enfermagem de Ribeirão Preto, Universidade de São Paulo.

06. FALEIROS SOUSA, F.A.E. Mensuração: escalas de categorias versus escalas de razão. Acta Paul. Enfermagem, 1999. (no prelo).

07. FALEIROS SOUSA, F.A.E.; Da SILVA, J.A. Psicofísica do prestígio social: comparação entre estimação de magnitudes e de comparação aos pares. Arq. Bras. Psicol., v. 48, p. 69-79, 1996a.

08. FALEIROS SOUSA, F.A.E.; Da SILVA, J.A. Uso e aplicação da metodologia psicofísica na pesquisa em enfermagem. Rev.latino-am.Enfermagem, v. 4, p. 147-78, 1996b.

09. FALEIROS SOUSA, F.A.E.; Da SILVA, J.A. Scaling of social prestige by direct and indirect psychophysical methods. Perceptual and Motor Skills, 1999a. (no prelo).

10. FALEIROS SOUSA, F.A.E.; Da SILVA, J.A. Validação da escala de razão de prestígio profissional do enfermeiro através do método de emparelhamento intermodal. Rev.latinoam.Enfermagem, v. 7, n. 1, p. 27-37, 1999b.

11. FALEIROS SOUSA, F.A.E.; KAMIZAKI, R.; SANT'ANA, R.P.M.; GIUNTINI, P.B. Mensuração da gravidade de quadros clínicos resultantes de cirurgias. Medicina, v. 31, n. 4, p. 616-625, 1998.

12. GUIRARDELLO, E.B. Factors in the critical care and medical-surgical environments that increase the requirements for directed attention. Madison, 1993. 75p. Master' thesis School of Nursing, University of Wisconsin United States.
13. HAMBLIM, R.L. Social attitudes, magnitude measurement and theory. In: BLALOCK Jr. H. (ed.). Measurement in the social sciences: theories and strategies. Chicago: Aldine Publishing, 1974. p. 61-120.

14. HINSHAW, A.S. Role attitudes: a measurement problem. In: HARDY, M.E.; CONWAY, M.E. (eds.). Role theory perspectives for health professionals. Michigan: Appleton - Century Crofts, 1978. p. 273-304.

15. HINSHAW, A.S.; FIELD, M.A. An investigation of variables that underlie collegial evaluation. Nurs. Res., v. 23, n. 4, p. 292-300, 1974.

16. HINSHAW, A.S.; OAKES, D. Theoretical modeltesting: patients' nurses' and physicians' expectations for quality nursing care. Commun. Nurs. Res., v. 10, p. 163-187, 1977.

17. JAMES, W. The principles of psychology. Cambridge, MA: Dover Publications, 1950. (Original inglês, 1890).

18. KAMIZAKI, R.; FALEIROS SOUSA, F.A.E.; Da SILVA, J.A. Validação da escala de razão de gravidade de quadros clínicos através do método de emparelhamento intermodal. Arq. Bras. Psicol., v. 50, n. 1/2, p. 72-91, 1998.

19. KAPLAN, S. A model of person-environment compatibility. Environ. Behav., v. 15, p. 311-332, 1983.

20. KAPLAN, S.; KAPLAN, R. Cognition and environment: functioning in an uncertain world. New York: Praeger, 1982. 287p.

21. LODGE, M. Magnitude scaling: quantitative measurement of opinions. Berverly Hills, CA: Sage, 1981. 87p.

22.MEEK, P.M.; SENNOTT-MILLER, L.; FERKETICH, S.L. Scaling stimuli with magnitude estimation. Res. Nurs. Health, v. 15, p. 77-81, 1992.

23. STEVENS, S.S. On the psychophysical law. Psychol. Rev., v. 64, n. 3, p. 153-181, 1957.

24. STEVENS, S.S. A metric for the social consensus. Science, v. 151, p. 530-541, 1966.

25. STEVENS, S.S. Psychophysics: introduction to its perceptual, neural and social prospects. New York: John Wiley, 1975.

26. STEVENS, S.S.; GALANTER, E.R. Ratio scales and category scales for a dozen perceptual continua. J. Exp. Psychol., v. 54, n. 6, p. 377-411, 1957.

Recebido em: 3.3.1999

Aprovado em: 7.12.1999 